\title{
Mott Transitions in Multiorbital Systems
}

\author{
A. Liebsch* \\ Institut für Festkörperforschung, Forschungszentrum Jülich, 52425 Jülich, Germany
}

(Received 14 July 2003; published 24 November 2003)

\begin{abstract}
Using the dynamical mean field theory it is shown that interorbital Coulomb interactions in nonisotropic multiorbital materials give rise to a single Mott transition. Nevertheless, narrow and wide subbands exhibit different excitation spectra in the metallic and insulating phases. The close analogy between "multigap" insulating behavior and multigap superconductivity is pointed out.
\end{abstract}

PACS numbers: 71.20.Be, 71.27.+a, 79.60.Bm

A hallmark of strongly correlated materials is their geometrical, electronic, and magnetic complexity [1]. Among the fascinating phenomena observed in these systems are metal-insulator transitions caused by local Coulomb interactions [2]. Valence bands of Mott insulators are mostly derived from oriented electronic orbitals whose degeneracy usually is lifted by the crystal field, giving rise to a variety of highly anisotropic properties. For instance, the layer-perovskite $\mathrm{Sr}_{2} \mathrm{RuO}_{4}$ consists of a wide, two-dimensional $d_{x y}$ band and narrow, nearly onedimensional $d_{x z, y z}$ bands $[3,4]$. This system exhibits unconventional $p$-wave superconductivity [5], but isoelectronic replacement of $\mathrm{Sr}$ by $\mathrm{Ca}$ induces a Mott transition to an antiferromagnetic insulator [6]. Similar splittings into nonequivalent subbands occur in the classic Mott insulators $\mathrm{VO}_{2}[7,8]$ and $\mathrm{V}_{2} \mathrm{O}_{3}[9,10]$, in layered organic superconductors [11], fullerenes [12], and many other compounds [1].

The nature of the paramagnetic metal-insulator transition in a multiband environment involving subbands of different widths is not yet understood. In particular, it is not clear whether all bands undergo a common transition at the same critical Coulomb energy, or whether narrow and wide subbands generate transitions at successive critical Coulomb energies. While local Coulomb interactions in multiband materials have been investigated previously [13-20], most studies treated the special "isotropic" case of identical orbitals where the question of one or several Mott transitions does not arise. Here we consider the "nonisotropic" case where the multiband system consists of nonequivalent subbands which is the usual situation in typical Mott insulators. In the absence of interorbital Coulomb interactions, and in the case of negligible hybridization, these bands would exhibit metal-insulator transitions at different critical energies $U_{c i}$.

Using the dynamical mean field theory [21], we show that the consequence of interorbital Coulomb interactions is to enforce a single Mott transition, implying all subbands to be either metallic or insulating. The critical Coulomb energy $U_{c}$ of the interacting system lies between the $U_{c i}$ of the isolated subbands. Thus, narrow subbands are less correlated and wide subbands are more correlated than in the absence of interorbital Coulomb interactions. Coexistence of metallic and insulating behavior in different subbands does not occur. On the other hand, in the joint metallic region narrow and wide subbands reveal excitation spectra with varying correlation signatures. Moreover, the insulating phase exhibits a "multigap" spectrum, i.e., the subbands show different excitation gaps and Hubbard bands.

This novel picture for multiband Mott insulators is remarkably similar to the "orbital-dependent" superconductivity observed in the quasi-two-dimensional compounds $\mathrm{Sr}_{2} \mathrm{RuO}_{4}$ [5] and $\mathrm{MgB}_{2}$ [22-25]. In the latter case, the intra- and interplanar electronic orbitals couple to anisotropic phonon modes and lead to different superconducting order parameters. Nevertheless, the weak hybridization interaction between the $\sigma$-and $\pi$-type orbitals ensures that this multigap material exhibits a single transition, i.e., both excitation gaps vanish at the same critical temperature.

Let us consider the paramagnetic metal-insulator transition in a two-band Hubbard model with narrow and wide subbands. These might represent, for instance, the $t_{2 g}$ orbitals of a layer-perovskite material or of $\mathrm{VO}_{2}$. Hybridization between subbands is assumed to be small, e.g., for symmetry reasons. For illustrative purposes these bands are assumed to be half filled and to have elliptical densities of states $\rho_{i}(\omega)$ of widths $W_{1}=2 \mathrm{eV}$ and $W_{2}=$ $4 \mathrm{eV}$. Single-band systems of this type have been investigated extensively in the past and their metal-insulator transitions are well understood [26]. To account for local Coulomb correlations we use the dynamical mean field theory (DMFT) in combination with the multiorbital quantum Monte Carlo (QMC) method [21]. The quantum impurity problem is solved at finite temperature $T$ for various intra- and interorbital Coulomb energies $U$ and $U^{\prime}=U-2 J$, where $J$ is the Hund's rule exchange integral.

Two criteria are employed to determine the stability of the Fermi liquid state as a function of the on-site Coulomb energy. First, we calculate the subband quasiparticle weights

$$
Z_{i}=\left[1-\partial \operatorname{Re} \Sigma_{i}(\omega) /\left.\partial \omega\right|_{\omega=0}\right]^{-1},
$$


where the derivative of the real part of the self-energy $\Sigma_{i}(\omega)$ is approximated by $\operatorname{Im} \Sigma_{i}\left(i \omega_{0}\right) / \omega_{0}$ with $\omega_{0}=$ $\pi / \beta=\pi k_{B} T$ being the first Matsubara frequency. $1 / Z_{i}$ defines the effective mass ratio of the $i$ th subband and $1-$ $Z_{i}$ provides the correlation induced transfer of spectral weight from the coherent peak at the Fermi level to the incoherent Hubbard bands. The second criterion is obtained from the imaginary-time Green's functions at $\tau=\beta / 2$ :

$$
G_{i}(\beta / 2)=\pi^{-1} \int d \omega F(\omega) \operatorname{Im} G_{i}(\omega)
$$

where $F(\omega)=0.5 / \cosh (\beta \omega / 2)$ is a distribution of width $w=4 \ln (2+\sqrt{3}) / \beta$ centered about the Fermi level. While $Z_{i}$ specifies the quasiparticle weight of the $i$ th subband right at $E_{F}, G_{i}(\beta / 2)$ represents the integrated spectral weight within a few $k_{B} T$ of $E_{F}$, i.e., it includes the low-lying excitations. Below we present normalized quantities $\bar{G}_{i}(\beta / 2)=G_{i}(\beta / 2) / G_{i}^{U=0}(\beta / 2)$ so that $Z_{i}=$ $\bar{G}_{i}(\beta / 2)=1$ in the noninteracting limit. Studying these criteria is convenient since $\Sigma_{i}\left(i \omega_{n}\right)$ and $G_{i}(\tau)$ follow directly from the QMC calculation. Thus, $Z_{i}$ and $\bar{G}_{i}(\beta / 2)$ are available without having to evaluate the real-frequency spectral distributions $\operatorname{Im} G_{i}(\omega)$.

Figure 1 shows the variation of $Z_{i}$ and $\bar{G}_{i}(\beta / 2)$ as a function of $U$ for $T=125 \mathrm{meV}$. The exchange integral is $J=0.2 \mathrm{eV}$ [27]. Evidently the quasiparticle weights near $E_{F}$ diminish as a result of Coulomb correlations. At a given $U$, however, $Z_{1}$ and $\bar{G}_{1}(\beta / 2)$ in the nonisotropic $W_{1}, W_{2}$ system are larger than in the isotropic $W_{1}, W_{1}$ case. Thus, as a result of interorbital interactions the narrow subband is less correlated than in the degenerate two-band system. Similarly, $Z_{2}$ and $\bar{G}_{2}(\beta / 2)$ in the $W_{1}, W_{2}$ model are smaller than in the $W_{2}, W_{2}$ case, i.e., interorbital interactions make the wide subband less metallic than in the isotropic two-band system.

Since $\bar{G}_{i}(\beta / 2)$ represents the spectral weight within several $k_{B} T$ of $E_{F}$ its reduction at small $U$ is weaker than that of $Z_{i}$, while in the critical regions its decay is more

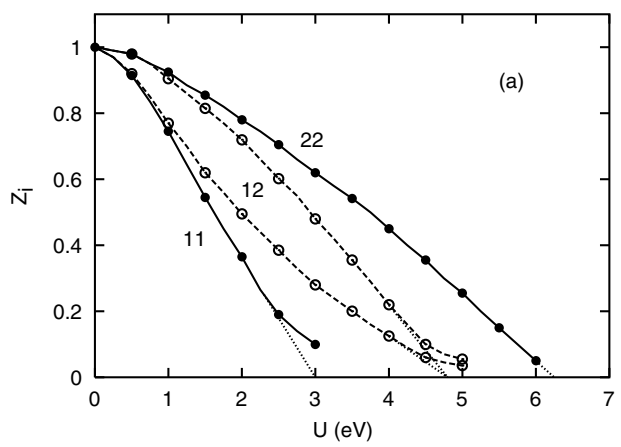

abrupt. Close to the metal-insulator transitions $Z_{i}$ and $\bar{G}_{i}(\beta / 2)$ show a characteristic rounding off caused by the finite temperature and critical slowing down. Within this uncertainty $Z_{i}$ and $\bar{G}_{i}(\beta / 2)$ yield consistent critical Coulomb energies: $U_{c 1} \approx 3.0 \mathrm{eV}$ for the narrow twoband system, $U_{c 2} \approx 6.1 \mathrm{eV}$ for the wide two-band system, and $U_{c} \approx 4.8 \mathrm{eV}$ for the mixed system [28].

The key point of these results is the fact that the system involving narrow and wide subbands exhibits a single Mott transition at an intermediate $U_{c}$ such that $U_{c 1}<$ $U_{c}<U_{c 2}$. There is no evidence for coexisting insulating and metallic subbands. All bands are either metallic or insulating [29]. At small $U$ correlations reduce the quasiparticle weight in the narrow subband much more rapidly than in the wide band. For $U>U_{c} / 2$, however, $Z_{1}$ and $Z_{2}$ begin to converge again and decrease to zero spectral weight at the same $U_{c}$. The same behavior is shown by the integrated weights $\bar{G}_{i}(\beta / 2)$. Since $Z_{1}<Z_{2}$ and $\bar{G}_{1}<\bar{G}_{2}$ the narrow band is always more strongly correlated than the wider counterpart. Analogously, the insulating phase exhibits two excitation gaps (see below).

The picture of a common Mott transition in a nonisotropic multiorbital environment is consistent with the general mean field theory of phase transitions in interacting subsystems [30]. It confirms our preliminary results for $\mathrm{Ca}_{2-x} \mathrm{Sr}_{x} \mathrm{RuO}_{4}$ [31]. These results were not entirely conclusive due to uncertainties associated with the maximum entropy [32] reconstruction of real-frequency spectral distributions from the QMC imaginary-time Green's functions. Here we avoid these difficulties by investigating more reliable criteria for the stability of the Fermi liquid state directly within imaginary time/frequency space. Also, by varying the Coulomb energy over a wide range, the evolution from noninteracting to strongly interacting subbands is clearly revealed. The present picture does not support the "orbital-selective" Mott transitions obtained for $\mathrm{Ca}_{2-x} \mathrm{Sr}_{x} \mathrm{RuO}_{4}$ by Anisimov et al. [19] nor the "two-fluid" metal-insulator scenario for $\mathrm{VO}_{2}$ found by Laad et al. [33]. At present the origin of these differences is not known [34].

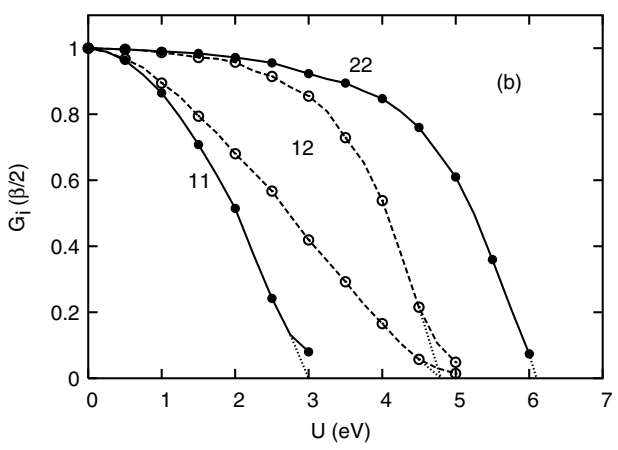

FIG. 1. (a) Quasiparticle weights $Z_{i}$ and (b) integrated spectral weights $\bar{G}_{i}(\beta / 2)$ of two-band systems as a function of Coulomb energy $U$ for $T>0$. Solid symbols: isotropic $W_{1}, W_{1}$ and $W_{2}, W_{2}$ systems; open symbols: nonisotropic $W_{1}, W_{2}$ system. Dotted lines: extrapolation of $Z_{i}$ and $\bar{G}_{i}(\beta / 2)$ to estimate critical Coulomb energies. The convergence of the dashed lines towards increasing $U$ indicates the existence of a single Mott transition in the nonisotropic two-band system. 


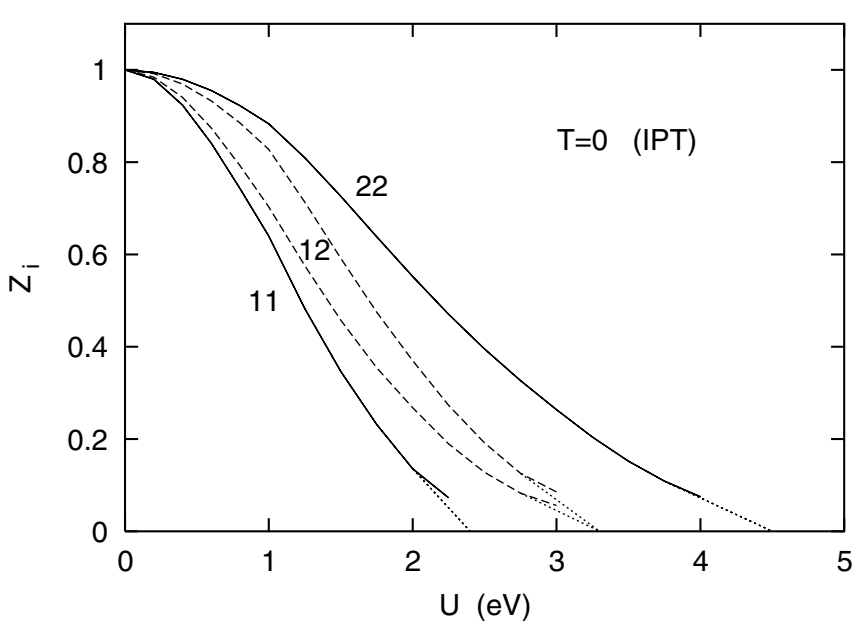

FIG. 2. Quasiparticle weights $Z_{i}$ of two-band systems as a function of Coulomb energy $U$ for $T=0$. Solid curves: isotropic $W_{1}, W_{1}$ and $W_{2}, W_{2}$ systems; dashed curves: nonisotropic $W_{1}, W_{2}$ system. Dotted lines: extrapolation of $Z_{i}$ to estimate critical Coulomb energies. As in Fig. 1, the convergence of the dashed lines towards increasing $U$ signifies the existence of a single Mott transition in the nonisotropic two-band system.

Multiband QMC calculations at lower temperatures $(T=62$ and $31 \mathrm{meV})$ confirm the picture discussed above. To verify that the main conclusion holds also in the low temperature limit we have performed analogous twoband calculations at $T=0$ within the self-consistent iterated perturbation theory (IPT) [35], where the selfenergy is calculated at real frequencies within the second-order perturbation theory. As shown in Fig. 2, the behavior of the quasiparticle weights at $T=0$ is consistent with the scenario for $T>0$ : The isotropic two-band systems have widely different critical Coulomb energies, $U_{c 1} \approx 2.3 \mathrm{eV}$ and $U_{c 2} \approx 4.3 \mathrm{eV}$. In contrast, the nonisotropic system undergoes a Mott transition at $U_{c} \approx 3.2 \mathrm{eV}$ [36].

Although interorbital Coulomb interactions lead to a common metal-insulator transition in the narrow and wide subbands, their excitation spectra differ qualitatively both in the metallic and insulating phases. This is illustrated in Fig. 3 which shows quasiparticle distributions derived using the maximum entropy method [32]. For $U<U_{c}$ both bands are metallic. The narrow band spectrum $N_{1}(\omega)$, however, is more strongly correlated than the wide band spectrum $N_{2}(\omega)$ : The spectral weight at $E_{F}$ is greatly reduced and the Hubbard peaks are more pronounced. In the absence of interorbital Coulomb interactions, $N_{1}(\omega)$ would exhibit a gap whereas $N_{2}(\omega)$ would be much less correlated than the spectral distribution shown in Fig. 3(a). For $U>U_{c}$ we find that the insulating phase is multigapped: both subbands exhibit excitation gaps, but the gap of the narrow band is larger than that of the wide band. In the absence of interorbital interactions the gap in $N_{1}(\omega)$ would be even larger, while according to Fig. 1 for $U=6 \mathrm{eV}, N_{2}(\omega)$ would be close to the metalinsulator transition.

For illustrative purposes we have studied the case of half-filled, symmetric subbands. The consistency of the picture discussed here with that for $\mathrm{Ca}_{2-x} \mathrm{Sr}_{x} \mathrm{RuO}_{4}$ [31] suggests that the same conclusion holds more generally for nonsymmetric multiband systems.

Our results provide new insight into the nature of Mott transitions in multiorbital systems. Several predictions can be tested using angle resolved photoemission and optical spectroscopy. In the metallic phase, the subbands should exhibit coherent peaks of different spectral weights and different incoherent satellite features. At the transition the coherent peaks should vanish simultaneously. In the insulating phase, the subbands should exhibit different excitation gaps and Hubbard bands. The gaps should close simultaneously upon approaching the transition.

In summary, we have studied the effect of interorbital Coulomb interactions on the metal-insulator transition of nonisotropic multiband materials. Using the dynamical mean field theory we have shown that narrow and wide subbands exhibit a common Mott transition rather than a
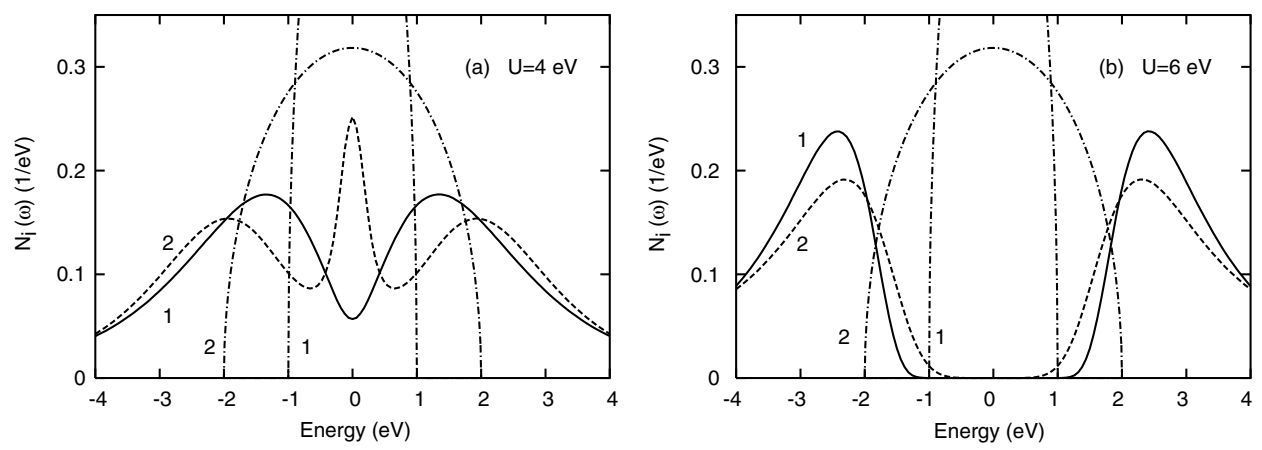

FIG. 3. Quasiparticle spectra of nonisotropic two-band system for $T=125 \mathrm{meV}\left(E_{F}=0\right)$ : (a) metallic phase, (b) insulating phase. The solid and dashed curves denote the spectra of the narrow and wide subbands, respectively. The bare densities of states are represented by the dot-dashed curves. In the metallic phase, both subbands exhibit different spectral weight near $E_{F}=0$ and different incoherent peaks. In the insulating phase, they show different excitation gaps. 
sequence of transitions. In practice, hybridization between subbands should also contribute to the occurrence of a single transition. $\mathrm{In}_{\mathrm{Sr}_{x}} \mathrm{RuO}_{4}$, for instance, replacing $\mathrm{Sr}$ by $\mathrm{Ca}$ leads to distortions of oxygen octahedra and to coupling between $\mathrm{Ru} t_{2 g}$ bands. Hybridization occurs also between $\mathrm{V} t_{2 g}$ orbitals in $\mathrm{VO}_{2}$ and $\mathrm{V}_{2} \mathrm{O}_{3}$. The sharp resistivity jumps observed in many Mott insulators provide experimental evidence for the correctness of our picture of a single transition in a multiband environment. Nevertheless, it would be of interest to study the characteristic multiorbital features of these materials, such as the multigap nature of the insulating phase and the joint disappearance of the coherent peaks on the metallic side of the transition. These experiments should elucidate the analogy between multigap insulating behavior and multigap superconductivity.

I am grateful to A. Bringer for discussions, and to O. Gunnarsson and D. Vollhardt for comments. I would also like to thank A. Lichtenstein for the DMFTQMC code.

*Email address: a.liebsch@fz-juelich.de

[1] M. Imada, A. Fujimori, and Y. Tokura, Rev. Mod. Phys. 70, 1039 (1998).

[2] N.F. Mott, Metal Insulator Transitions (Taylor and Francis, London, 1990).

[3] T. Oguchi, Phys. Rev. B 51, 1385 (1995).

[4] I. I. Mazin and D. Singh, Phys. Rev. Lett. 79, 733 (1997).

[5] Y. Maeno, T. M. Rice, and M. Sigrist, Phys. Today 54, No. 1, 42 (2001).

[6] S. Nakatsuji and Y. Maeno, Phys. Rev. Lett. 84, 2666 (2000).

[7] J. B. Goodenough, Phys. Rev. 117, 1442 (1960).

[8] A. Zylbersztejn and N. F. Mott, Phys. Rev. B 11, 4383 (1975).

[9] M. J. Rozenberg, G. Kotliar, H. Kajueter, G. A. Thomas, G. H. Rapkine, J. M. Honig, and P. Metcalf, Phys. Rev. Lett. 75, 105 (1995).

[10] S. Yu. Ezhov, V. I. Anisimov, D. I. Khomskii, and G. A. Sawatzky, Phys. Rev. Lett. 83, 4136 (1999).

[11] S. Lefebvre et al., Phys. Rev. Lett. 85, 5420 (2000).

[12] T. Takenobu, T. Muro, Y. Iwasa, and T. Mitani, Phys. Rev. Lett. 85, 381 (2000).

[13] O. Gunnarsson, E. Koch, and R. M. Martin, Phys. Rev. B 54, R11026 (1996).

[14] A. Liebsch and A. I. Lichtenstein, Phys. Rev. Lett. 84, 1591 (2000).

[15] K. Held et al., in Quantum Simulations of Complex Many-Body Systems: From Theory to Algorithms, edited by J. Grotendorst, D. Marks, and A. Muramatsu, NIC Series Vol. 10 (John von Neumann Institute for
Computing, Jülich, 2002), p. 175. See also references herein.

[16] N. Manini, G. Santoro, A. Dal Costa, and E. Tosatti, Phys. Rev. B 66, 115107 (2002).

[17] A. Koga, Y. Imai, and N. Kawakami, Phys. Rev. B 66, 165107 (2002).

[18] S. Florens, A. Georges, G. Kotliar, and O. Parcollet, Phys. Rev. B 66, 205102 (2002).

[19] V. I. Anisimov, I. A. Nekrasov, D. E. Kondakov, T. M. Rice, and M. Sigrist, Eur. Phys. J. B 25, 191 (2002).

[20] Y. Ōno, M. Potthoff, and R. Bulla, Phys. Rev. B 67, 035119 (2003).

[21] For a review, see A. Georges, G. Kotliar, W. Krauth, and M. J. Rozenberg, Rev. Mod. Phys. 68, 13 (1996).

[22] P. C. Canfield and G.W. Crabtree, Phys. Today 56, No. 3, 34 (2003).

[23] S. Suoma et al., Nature (London) 423, 65 (2003).

[24] A. Y. Liu, I. I. Mazin, and J. Kortus, Phys. Rev. Lett. 87, 087005 (2001).

[25] H. J. Choi, D. Roundy, H. Sun, M. L. Cohen, and S. G. Louie, Nature (London) 418, 758 (2002).

[26] See R. Bulla, T. A. Costi, and D. Vollhardt, Phys. Rev. B 64, 045103 (2001), and references therein.

[27] Below $U=1 \mathrm{eV}$, we assume $J \sim U$ so that $U^{\prime}$ remains positive. Other choices, such as $J=0, U^{\prime}=U$ or $J=$ $0.2 U, U^{\prime}=0.8 U$, do not differ qualitatively from the results shown in Figs. 1 and 2.

[28] $U_{c 1}$ and $U_{c 2}$ do not differ exactly by a factor of 2 since $\beta$ and $J$ are kept constant. Only if all quantities are changed by the same factor $U_{c}$ scales accordingly.

[29] As shown in Ref. [18] for the isotropic case, at very low $T$ the insulating phase begins at a $U_{c}^{\prime}$ slightly below $U_{c}$ denoting the disappearance of the metallic phase. Similar behavior might occur for nonisotropic multiorbital systems, with a common $U_{c}^{\prime}<U_{c}$ for all subbands. This low temperature region is computationally not yet accessible to multiorbital DMFT-QMC calculations.

[30] K. Binder, in Phase Transitions and Critical Phenomena, edited by C. Domb and J.L. Lebowitz (Academic, London, 1983), Vol. 8.

[31] A. Liebsch, Europhys. Lett. 63, 97 (2003).

[32] M. Jarrell and J. E. Gubernatis, Phys. Rep. 269, 133 (1996).

[33] M. S. Laad, L. Craco, and E. Müller-Hartmann, condmat/0305081.

[34] Recent work by the authors of Ref. [19] agrees with the present picture: V. I. Anisimov (private communication).

[35] A. Georges and G. Kotliar, Phys. Rev. B 45, 6479 (1992).

[36] These energies differ from those in Fig. 1 since the IPT implies a more approximate treatment of correlations and because of the different temperatures. 